\title{
Successful Abdominal Organ Donation after Brain Death in a Patient with a Biventricular Assist Device: Extending Extended Criteria
}

\author{
Susanne Carpenter ${ }^{1}$, D. Eric Steidley ${ }^{2}$, David D. Douglas ${ }^{3}$, K. Sudhakar Reddy ${ }^{1}$, David Mulligan ${ }^{1}$, \\ Louis Lanza ${ }^{4}$, Adyr Moss ${ }^{1}$ \\ ${ }^{1}$ Division of Transplant Surgery, Mayo Clinic Arizona, Phoenix, USA \\ ${ }^{2}$ Division of Cardiovascular Diseases, Mayo Clinic Arizona, Phoenix, USA \\ ${ }^{3}$ Division of Hepatology, Mayo Clinic Arizona, Phoenix, USA \\ ${ }^{4}$ Divsion of Cardiothoracic Surgery, Mayo Clinic Arizona, Phoenix, USA \\ Email: carpenter.susanne@mayo.edu
}

Received December 1, 2012; revised January 4, 2013; accepted January 12, 2013

Copyright (c) 2013 Susanne Carpenter et al. This is an open access article distributed under the Creative Commons Attribution License, which permits unrestricted use, distribution, and reproduction in any medium, provided the original work is properly cited.

\begin{abstract}
Few studies address the potential for donation after brain death (DBD) in the limited population of patients with ongoing mechanical circulatory support (MCS). A case study was conducted reviewing available records of both donor and recipient, and available literature. The donor was a young female with an acute myocardial infarction precipitating emergent off-pump 2-vessel bypass graft complicated by profound cardiogenic shock refractory to inotropes and intra-aortic balloon pump. A heparin drip was started following percutaneous placement of a left ventricular-assist device $\left(\right.$ TandemHeart ${ }^{\circledR}$ ) which improved her hemodynamics to stabilize for transfer. She ultimately required surgical placement of biventricular assist device $\left(\mathrm{CentraMag}^{\circledR}\right)$ to normalize hemodynamics. Two days post-operatively, she developed a cerebellar hemorrhage and was declared brain dead. Pre-donation blood chemistry showed adequate end-organ function. Both kidneys were placed locally. The liver was rejected for two regional status 1 patients and by all other local centers. We accepted the liver for a patient with polycystic liver disease with a MELD exception score of 20. The recipient is now 4 years post-transplant with excellent graft function. Extending donor criteria to include MCS patients can result in successful transplantation and should be considered in selected circumstances once satisfactory donor end-organ function is established.
\end{abstract}

Keywords: Extended Criteria Organ Donation; Donation after Cardiac Death; Ventricular Assist Device

\section{Introduction}

The demand for suitable transplant organs exceeds the available supply both in the United States and throughout the world. With the growth of transplant waitlists, criteria for organ donation are being extended to include older and more ill donors [1]. Gradually, what was once considered as a marginal donor is now often accepted as extended criteria gains widespread acceptance [1,2].

Cardiac and cardio-pulmonary mechanical circulatory support systems including extracorporeal membrane oxygenation (ECMO) and various ventricular assist devices (VADs) are increasingly employed in the United States to support critically ill patients to restore end-organ function during cardiogenic shock [3]. While the last ten years have seen substantial advances in the field, mechanical circulatory support (MCS) systems are still fraught with potential complications like hemorrhagic and embolic cerebrovascular accidents, driveline infections, component failure, and long-term end-organ dysfunction [4,5]. Patients suffering catastrophic cerebral complications while on MCS present the potential donor. At least two uses of ECMO as a facilitator of organ perfusion prior to organ recovery have been reported $[6,7]$. Similarly, patients with MCS may achieve optimal endorgan perfusion via device flow manipulation even after brain death is declared. At least one donation has been reported from a patient who was undergoing outpatient VAD bridge-to-transplant therapy and became a donor candidate following presentation with acute cerebrovascular accident (CVA) and brain death [8]. Many centers have performed procurements of this nature with patients as a donation after cardiac death (DCD) which have gone 
unreported.

This study reports the procurement of liver and kidneys as a donation after brain death from a donor who suffered acute cardiogenic shock, was maintained on continuous flow bilateral ventricular assist device (BiVAD) support, and then suffered a hemorrhagic CVA which precipitated brain death. A literature review is performed and other reports of similar procurements are summarized, and the potential for future employment of donors sustained via MCS is discussed.

\section{Results}

\subsection{Donor Procurement}

The donor was a 47-year-old female smoker who suffered an acute large anterior wall myocardial infarction. An emergent off-pump two-vessel coronary bypass was performed at an outside hospital. Post-operatively the patient developed cardiogenic shock refractory to inotropes and intra-aortic balloon pump. A percutaneously placed LVAD (TandemHeart ${ }^{\circledR}$, CardiacAssist, Inc., Pittsburgh, PA) improved her hemodynamics and stabilized her for transfer to our facility, but significant hypotension and depressed cardiac output persisted despite inotropes and vasopressors. She was placed on heparin infusion. She was transferred to our institution where a surgical biventricular assist device (CentraMag ${ }^{\circledR}$, Thoratec Corporation, Pleasanton, CA) was placed using cardiopulmonary bypass with cannulation on from right ventricle to pulmonary artery and from left ventricle to aorta. Hemodynamics improved and the patient weaned off inotropic and vasopressor support promptly. Two days post-operatively the patient developed bradycardia with fixed and dilated pupils. A computed tomography (CT) with angiogram of the head showed an intracranial hemorrhage and she was declared brain dead based on standard criteria-which included the aforementioned perfusion study as well as apnea testing, and the absence of corneal and gag reflexes, and the presence of blown pupils. She was approved for liver and kidney donation and the family was consented for organ donation. Predonation blood chemistry showed creatinine of 0.7 , AST 88, ALT 21, INR 1.46, and $\mathrm{pH}$ of 7.33 and $\mathrm{paO}_{2}$ 85.3. Both kidneys were placed locally. The liver was turned down for two regional Status 1 patients and by all other local centers.

The donor's chest was open since BiVAD placement (Figure 1), and the donor was transferred to the operating room and supported on the biventricular assist device (BiVAD) until cold histidine-tryptophane-ketogluatarate (HTK) was infused. Due to the amount of space occupied by the BiVAD equipment, a xiphoid to pubis midline incision was utilized. Gross appearance of the liver was felt to be satisfactory. Major vessels were isolated, and

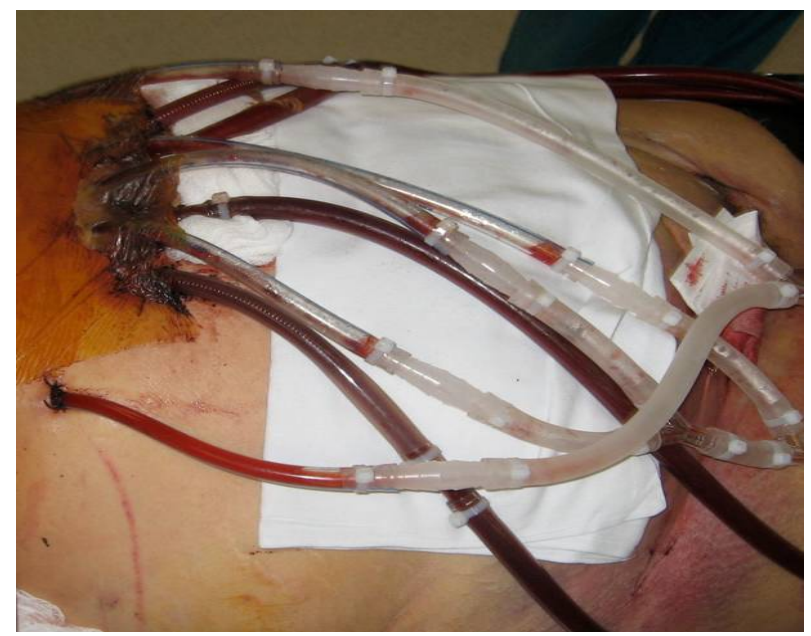

Figure 1. Pre-operative donor with open chest.

aorta and portal vein were cannulated. The aorta was cross-clamped, the suprahepatic vena cava was divided and rapid portal and aortic infusion of HTK was initiated. The BiVAD was then stopped. 2 liters of portal flush and 4 liters of aortic flush were utilized in conjunction with ice and the flush was performed with ease. After flushing was completed, hepatectomy and bilateral nephrectomy were undertaken and iliac vessels were procured. No back-table flushing was performed. The liver anatomy was normal. Histologically, mild preservation injury was present without steatohepatitis, hepatic venous congestion, fibrosis, or inflammatory changes in portal tracts.

\subsection{Recipient Outcomes}

Our transplant team accepted the liver for a 44 year old female patient with massively enlarged liver due to polycystic liver disease with significant inhibition of her quality of life. She had been listed for six years with a native model for end-stage liver disease score (MELD) of 6. Just over six months prior to transplantation, the patient had been granted MELD exception points by the Regional Review Board and carried a MELD exception score of 20 at the time of transplantation. The patient was educated in detail about the unorthodox nature of the donor and possible consequences, and she agreed to proceed. Transplant hepatectomy and orthotopic liver transplantation were performed (Figure 2). Initially, a piggyback technique was attempted but the size of the native liver precluded this procedure. Thus the native supra- and infra-hepatic were isolated and divided upon hepatictomy. The native liver weighed $15 \mathrm{~kg}$ upon extraction (Figure 3). The donor suprahepatic vena cava was shortened for adequate length and anastomosed in an end-to-end fashion with a cloaca interconnection of all three hepatic veins. Similarly, the infrahepatic donor vena cava was anastomosed in an end-to-end fashion 


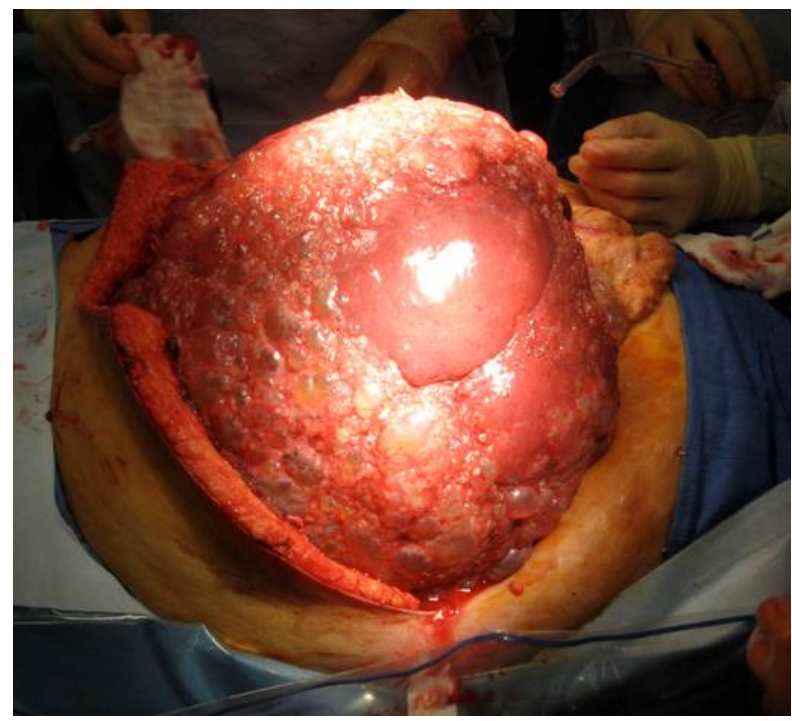

Figure 2. Intraoperative recipient hepatectomy.

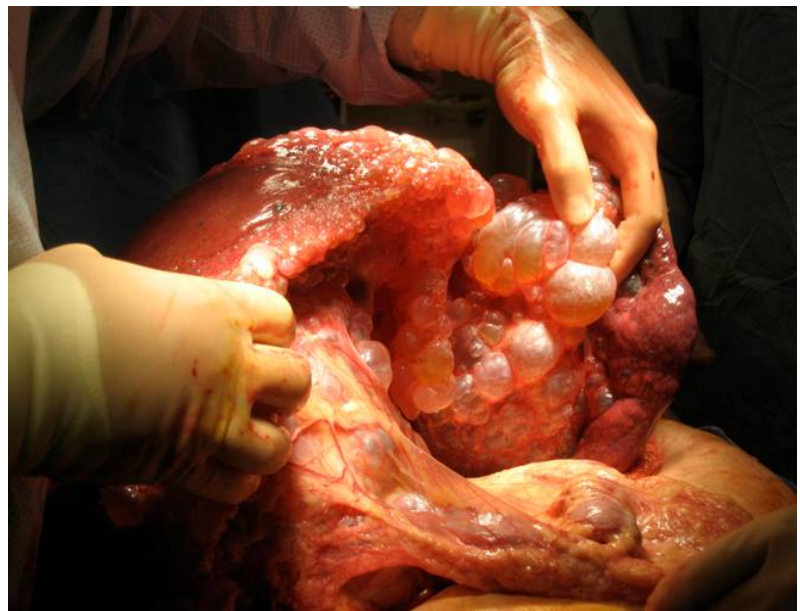

Figure 3. Intraoperative recipient hepatectomy.

with the recipient inferior vena cava (IVC) in an end-toend fashion using 4-0 prolene. Portal vein, hepatic artery, and common bile duct were all anastomosed in an endto-end fashion as well. Total cold ischemia time for the donor liver was 4.5 hours. Abdominal appearance was substantially improved at procedure's end (Figure 4).

The patient's post-operative course was relatively uneventful. Peak graft function laboratory tests were as follows: AST 1441, ALT 837, total bilirubin 3.4, direct bilirubin 2.0, PT 19.5, all of which occurred on postoperative day one and began resolving rapidly. Immunosuppression was achieved with CellCept, Prograf, and standard steroid taper. The peri-operative course was uneventful and the recipient is now more than 4 years post-transplant with excellent graft function.

Of note, both kidneys were accepted by local transplantation centers. They were functioning well per out side report at last follow-up. Whether or not a pump was

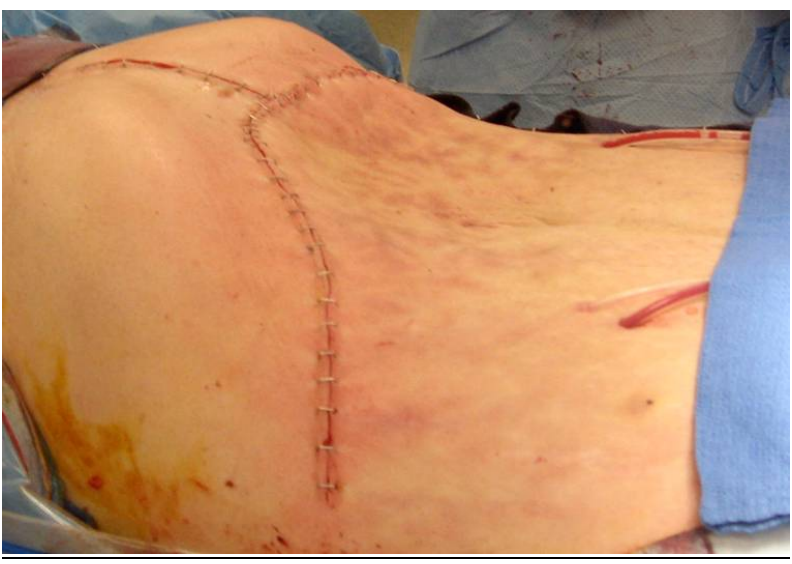

Figure 4. Post-operative recipient appearance.

utilized to facilitate delayed implantation is not known but kidneys were not immediately placed on pump at our institution secondary to satisfactory end-organ function.

\section{Discussion}

The successful utilization of organs procured from donors sustained with mechanical circulatory support (MCS) has been sparsely explored in transplant literature. In fact, while we are aware that this practice has taken place a number of institutions, an extensive literature review located only three similar case reports-all of which describe different methods of circulatory support maintenance and withdrawal in the peri-donation period (Table 1).

This donor would be considered marginal by most of the many proposed scoring systems for marginal liver donors, given her hypotension precipitated by cardiogenic shock that required substantial doses of inotropes and vasopressors $[2,9]$. However, given the lack of endorgan damage and an increasing comfort level with an increasing number of MCS-sustained patients at our institution, the transplant team at our institution felt comfortable accepting this liver for a recipient who otherwise would possibly still be awaiting transplant. The authors feel that this case supports the use of MCS donors, or at least a more serious consideration of them before unilateral rejection. In many cases their severe clinical histories can lead transplant teams to reject organs with satisfactory end-organ function.

The literature regarding use of marginal donors is conflicting. While some authors feel that marginal donor use portends no impact on recipient survival and graft function [10], others feel that especially in high-MELD donors, the risk of early post-transplant mortality is increased with marginal graft use [11]. Many feel that recipient status should be considered in the use of marginal donors, but the majority of publications advocate the use of marginal or extended donor criteria grafts when the 
Table 1. Donation \& mechanical circulatory support literature review.

\begin{tabular}{|c|c|c|c|}
\hline Author/Institution/Year & Donor & Support of Type & Method of Donation \\
\hline $\begin{array}{l}\text { Wang C. C. et al. } \\
\text { Kaohsiung Medical Center, Taiwan } \\
2005\end{array}$ & $\begin{array}{c}22 \text { yoF irreversible brain injury s/p } \\
\text { MVC and cardiac arrest }\end{array}$ & $\begin{array}{l}\text { VA ECMO-instituted after brain } \\
\text { death hypothermic } 5^{\circ} \mathrm{C}\end{array}$ & ECMO d/c'd prior to organ retrieval \\
\hline $\begin{array}{l}\text { Rayhill S. C. et al. } \\
\text { University of Iowa } \\
\text { Iowa City, IA } \\
2003\end{array}$ & $\begin{array}{l}52 \text { yoM with previous LVAD for } \\
\text { DCM presented with acute CVA }\end{array}$ & LVAD & $\begin{array}{l}\text { Prepped/Draped \& heparinized, } \\
\text { support withdrawn in OR, } \\
\text { then retrieval }\end{array}$ \\
\hline $\begin{array}{c}\text { Johnson L. B. et al. } \\
\text { University of Maryland } \\
\text { Baltimore, MD } \\
1997\end{array}$ & $\begin{array}{l}15 \text { yoM ARDS, respiratory failure, } \\
\text { ECMO } \times 29 \text { days, anoxic brain death } \\
\text { from circuit malfunction }\end{array}$ & VV then VA ECMO-normothermic & $\begin{array}{l}\text { ECMO continued via femoral } \\
\text { vessels until cold preservation }\end{array}$ \\
\hline
\end{tabular}

donor quality can be adapted for the proper recipients $[12,13]$. Regardless of the controversy in the literature, marginal donors are finding increased acceptance especially where age is concerned [1,2].

In conclusion, successful organ procurement from donors sustained via MCS has been reported in both a donation after cardiac death (DCD) setting and in this case of a brain dead donor. MCS donors with adequate endorgan function deserve the same consideration as other marginal donors and could help bridge the growing gap between organ supply and demand.

\section{REFERENCES}

[1] R. W. Busuttil and K. Tanaka, "The Utility of Marginal Donors in Liver Transplantation," Liver Transplantation, Vol. 9, No. 7, 2003, pp. 651-663. doi:10.1053/jlts.2003.50105

[2] S. G. Tullius, H. D. Volk and P. Neuhaus, "Transplantation of Organs from Marginal Donors,” Transplantation, Vol. 72, No. 8, 2001, pp. 1341-1349. doi:10.1097/00007890-200110270-00001

[3] C. B. Patel, K. M. Alexander and J. G. Rogers, “Mechanical Circulatory Support for Advanced Heart Failure," Current Treatment Options in Cardiovascular Medicine, Vol. 12, No. 6, 2010, pp. 549-565. doi:10.1007/s11936-010-0093-6

[4] R. C. Bogaev, S. V. Pamboukian, S. A. Moore, et al., "Comparison of Outcomes in Women versus Men Using a Continuous-Flow Left Ventricular Assist Device as a Bridge to Transplantation," The Journal of Heart and Lung Transplantation, Vol. 30, No. 5, 2011, pp. 515-522. doi:10.1016/j.healun.2010.12.009

[5] R. John, F. Kamdar, K. Liao, et al., "Improved Survival and Decreasing Incidence of Adverse Events with the Heart Mate II Left Ventricular Assist Device as Bridgeto-Transplant Therapy,” The Annals of Thoracic Surgery,
Vo. 86, No. 4, 2008, pp. 1234-1235.

doi:10.1016/j.athoracsur.2008.06.030

[6] C. C. Wang, S. H. Wang, C. C. Lin, et al., "Liver Transplantation from an Uncontrolled Non-Heart-Beating Donor Maintained on Extracorporeal Membrane Oxygenation,” Transplant Proceedings, Vol. 37, No. 10, 2005, pp. 4331-4333. doi:10.1016/j.transproceed.2005.11.013

[7] L. B. Johnson, J. S. Plotkin, C. D. Howell, et al., "Successful Emergency Transplantation of a Liver Allograft from a Donor Maintained on Extracorporeal Membrane Oxygenation,” Transplantation, Vol. 63, No. 6, 1997, pp. 910-911. doi:10.1097/00007890-199703270-00021

[8] S. C. Rayhill, G. Martinez-Mier, D. A. Katz, et al., "Successful Non-Heart-Beating Donor Organ Retrieval in a Patient with a Left Ventricular Assist Device," American Journal of Transplantation, Vol. 4, No. 1, 2004, pp. 144146. doi:10.1046/j.1600-6143.2003.00280.x

[9] J. Briceno, G. Solorzano and C. Pera, "A Proposal for Scoring Marginal Liver Grafts,” Transplant International, Vol. 13, Suppl. 1, 2000, pp. S249-S252. doi:10.1111/j.1432-2277.2000.tb02029.x

[10] G. Tisone, T. M. Manzia, S. Zazza, et al., "Marginal Donors in Liver Transplantation,” Transplant Proceedings, Vol. 36, No. 3, 2004, pp. 525-526. doi:10.1016/j.transproceed.2004.02.022

[11] T. Bacchella, F. H. Galvao, J. L. J. de Almeida, et al., "Marginal Grafts Increase Early Mortality in Liver Transplantation,” São Paulo Medical Journal, Vol. 126, No. 3, 2008, pp. 161-165. doi:10.1590/S1516-31802008000300005

[12] J. F. Renz, C. Kin, M. Kinkhabwala, et al., "Utilization of Extended Donor Criteria Liver Allografts Maximizes Donor Use and Patient Access to Liver Transplantation,” Annals of Surgery, Vol. 242, No. 4, 2005, pp. 556-563.

[13] M. Gastaca, "Extended Criteria Donors in Liver Transplantation: Adapting Donor Quality and Recipient," Transplant Proceedings, Vol. 41, No. 3, 2009, pp. 975979. doi:10.1016/j.transproceed.2009.02.016 